\title{
Gestão da cadeia de suprimentos: análise do desempenho ambiental de fornecedores verdes por meio do método fuzzy TOPSIS
}

\author{
Supply chain management: analysis of environmental performance of green suppliers by means \\ of the fuzzy TOPSIS method
}

\begin{abstract}
Resumo
Há um grande interesse por parte das organizações pela adoção de práticas sustentáveis, visando melhorar o desempenho de suas cadeias de suprimentos. Regulamentos ambientais, consumo de recursos e descarte de resíduos são alguns dos fatores que podem influenciar na tomada de decisão quanto a contratação de fornecedores verdes. Este estudo ilustra o caso de um fabricante de implementos agrícolas do setor metal mecânico que busca implementar o gerenciamento da cadeia de suprimentos verdes (GSCM) e selecionar um fornecedor que atenda aos requisitos ambientais determinados. Portanto, o objetivo desta pesquisa consiste em avaliar o desempenho ambiental de três potenciais fornecedores por meio do método de tomada de decisão multicritério fuzzy TOPSIS e selecionar a melhor alternativa. Além disso, é realizada uma análise de sensibilidade para comprovar a robustez do método utilizado. O resultado mostra que o fornecedor $\mathrm{A}_{3}$ ocupa o primeiro lugar entre os três fornecedores avaliados, demonstrando um forte desempenho ambiental, sobretudo, em quatro critérios: parceiros ambientais, imagem verde, reciclagem e descarte de resíduos.
\end{abstract}

Palavras-chave: GSCM; Fornecedores verdes; Desempenho ambiental; Fuzzy TOPSIS

\begin{abstract}
There is great interest from organizations in adopting sustainable practices to improve the performance of their supply chains. Environmental regulations, resource consumption and waste disposal are some of the factors that can influence decision making regarding the contracting of green suppliers. This study illustrates the case of a metalworking agricultural implements manufacturer looking to implement green supply chain management (GSCM) and select a supplier that meets specific environmental requirements. Therefore, the objective of this research is to evaluate the environmental performance of three potential suppliers through the TOPSIS fuzzy multicriteria decision-making method and to select the best alternative. In addition, a sensitivity analysis is performed to prove the robustness of the method used. The result shows the supplier $A_{3}$ occupies the first place among the best sellers, demonstrating a strong environmental performance, especially in four criteria: environmental partners, green image, recycling and waste disposal.
\end{abstract}

Keywords: GSCM; Green suppliers; Environmental performance; Fuzzy TOPSIS

Recebido: 12/09/2017 Aceito: 05/10/2017

Bruno Miranda ${ }^{1}$, Cyro Prato Neto 2 , Jovani Patias ${ }^{3}$, Alexandre Ferreira ${ }^{4}$, Leoni Godoy ${ }^{5}$

${ }^{1}$ Mestrando em Engenharia de Produção pela Universidade Federal de Santa Maria - UFSM - brmiranda10@ gmail.com

${ }^{2}$ Mestrando em Engenharia de Produção pela Universidade Federal de Santa Maria - UFSM - cyroprato@ yahoo.com.br

${ }^{3}$ Mestre em Engenharia de Produção pela Universidade Federal de Santa Maria - UFSM - jovanipatias@ gmail.com

${ }^{4}$ Doutorando em Engenharia de Produção pela UNISINOS - alexandreferreira.rs@ gmail.com

${ }^{5}$ Prof $^{a}$. Dr ${ }^{a}$. Do Programa de Pós-Graduação em Engenharia de Produção - UFSM - leonigodoy@ gmail.com 


\section{Introdução}

$\mathrm{U}$ $m$ dos principais objetivos da sociedade moderna é aliar o conhecimento sustentável com as problemáticas ambientais que fazem parte da atual realidade, estabelecendo uma sinergia entre as companhias e as responsabilidades de atuação no mercado, baseadas em metodologias de gestão voltadas para o desenvolvimento sustentável (DE CARVALHO; BARBIERI, 2013; SHEN et al., 2013). Tal fato evidencia o reflexo dos resultados positivos econômicos e sociais em políticas sustentáveis realizadas pelas empresas, para o alcance de vantagens competitivas de mercado.

A conscientização industrial para políticas ambientais teve seu início antes da década de 1980 , com inciativas para redução de resíduos, consumo de energia e materiais tóxicos. Após, foram desenvolvidas tecnologias limpas, com a finalidade de diminuir o impacto ambiental nas etapas de produção, atuando diretamente no processo. Posteriormente, na década de 1990, a conscientização ambiental se voltou na fase de projetos de produtos e serviços, introduzindo desenhos ecológicos (ANDIÇ; YURT; BALTACIOĞLU, 2013; SHEN et al., 2013). Atualmente, políticas de melhorias são executadas nos processos produtivos utilizando tecnologias limpas e reengenharia nos processos de fabricação, assim como o desenvolvimento de políticas ambientais nas indústrias de fornecedores da cadeia de suprimentos.

A utilização de critérios baseados na sustentabilidade para a seleção de fornecedores, incentivam as empresas que estão dentro da cadeia de suprimentos a adotarem políticas ambientais, como certificações e a implementação de práticas verdes (SHEN et al., 2013). A medida que evoluiu a conscientização para tais práticas, é natural a busca pela aquisição de produtos ou serviços que ofereçam baixo custo, alta qualidade e conformidade com padrões ambientais. Deste modo, para que ocorra a transformação desejada pelas empresas no desenvolvimento de produtos e processos, é necessária a relação direta com uma adequação dos fornecedores ambientalmente aptos. Segundo Zadeh (1965), a teoria dos conjuntos fuzzy é proposta para trabalhar com a imprecisão do pensamento humano na tomada de decisão, sendo identificado sua melhor utilização quando se aborda equidade e satisfação dos respondentes, vindo a fortalecer a compreensão dos resultados.

Para a seleção de fornecedores da cadeia produtiva, baseando-se em questões que englobam a sustentabilidade, utiliza-se o método de preferência por ordem de similaridade à solução ideal (TOPSIS). Desenvolvido por Hwang e Yoon (1981), esta metodologia é muito reconhecida para tomada de decisões multicritérios, uma vez que integra fatores qualitativos e quantitativos. Sua utilização é amplamente reconhecida, pois é facilmente desenvolvida em processos computacionais, na utilização de uma solução ideal e não ideal para se obter uma tendência de resposta, por meio da análise dos dados obtidos (CHEN; LIN; HUANG, 2006; JUNIOR; OSIRO; CARPINETTI, 2014).

Consta na literatura pesquisada, estudos desenvolvidos com a abordagem multicritério fuzzy para seleções de fornecedores, como a pesquisa de Shen et al. (2013), artigo que propõem uma abordagem fuzzy, baseada na green supply chain management (GSCM), que avalia fornecedores verdes, executando a metodologia fuzzy para compilar as percepções humanas linguísticas que, combinadas com a método TOPSIS geram uma pontuação global para cada fornecedor. Os estudos com a utilização da metodologia fuzzy TOPSIS nos últimos anos são investigados por diversos autores, como por exemplo: Awasthi, Chauhan e Goyal (2010); Gupta e Barua (2017); Dubey et al. (2015); e Luthra et al. (2016).

Gupta e Barua (2017) direcionaram seu estudo para a seleção de fornecedores entre pequenas e médias empresas, avaliando sua capacidade de inovação verde. Dubey et al. (2015) apontaram na literatura quatro influências na adoção de práticas sustentáveis na cadeia de suprimento verde, indicando seus facilitadores, assim como métodos mistos de maior utilização, realizaram também uma modelagem matemática para estruturar o índice de utilização dos métodos e por fim o nível de impacto do comprometimento organizacional da empresa na adoção de práticas para a GSCM. Luthra et al. (2016) forneceram em seus estudos avaliações comparativas das práticas de GSCM em empresas de fabricação de componentes automotivos da Índia, utilizando a técnica de TOPSIS difuso aplicado para avaliações comparativas para classificar as empresas selecionadas, teste realizado em quatro empresas.

O restante deste artigo subdivide-se em: seção 2, referencial teórico, na seção 3 é apresentada a 
metodologia abordada e o desenvolvimento da pesquisa. Dentro da seção 4, evidenciam-se os resultados e a seção 5 é exposto a conclusão.

\section{Referencial teórico}

O Sistema de Gestão Ambiental (SGA) é considerado uma abordagem para o cumprimento da regulamentação ambiental (EL HOUR et al., 2016). Inerente a isso, requisitos legais, controle operacional, avaliação de desempenho, entre outros (MAZZI et al., 2016). Pode-se nomear a ISO 14000 e BS 7750, como alguns dos SGA adotados por empresas para adequarem-se aos requisitos ambientais impostos (SHEN et al., 2012).
A vantagem de possuir um SGA pode gerar, de forma personalizada e consistente, uma abordagem para o entendimento do monitoramento ambiental, acerca de diversos âmbitos (KHALILI; DUECKER, 2013). Analisando-se a literatura da área, percebe-se a presença de estudos relacionados à preocupação ambiental atrelada à seleção de fornecedores. No estudo de Shen et al. (2012), foram pesquisadas referências para avaliação do desempenho ambiental de fornecedores, totalizando 24 estudos, no período de 1998 a 2012. A Tabela 1 busca completar a pesquisa bibliográfica supracitada.

\section{Tabela 1 - Avaliação do desempenho ambiental de fornecedores}

\begin{tabular}{c|c}
\hline Resumo dos artigos & Referências \\
\hline $\begin{array}{c}\text { Abordar a diferença no processo de seleção de fornecedores verdes em 3 } \\
\text { diferentes indústrias de eletrônicos na China }\end{array}$ & $\begin{array}{c}\text { Chiou et al. } \\
(2008)\end{array}$ \\
\hline $\begin{array}{c}\text { Analisar a performance dos critérios de seleção (entre eles o SGA), para } \\
\text { posterior implementação de um modelo fuzzy-AHP para avaliação dos } \\
\text { fornecedores verdes }\end{array}$ & $\begin{array}{c}\text { Grisi et al. } \\
(2010)\end{array}$ \\
\hline $\begin{array}{c}\text { Considerar o fator ambiental na aplicação da abordagem da teoria dos } \\
\text { conjuntos fuzzy e análise relacional grey para seleção dos fornecedores } \\
\text { de uma indústria de eletrônicos }\end{array}$ & $\begin{array}{c}\text { Chen et al. } \\
(2010)\end{array}$ \\
\hline $\begin{array}{c}\text { Avaliação e seleção de fornecedores verdes usando o método VIKOR } \\
\text { embutido em um sistema especializado fuzzy com números difusos com de intervalo }\end{array}$ & $\begin{array}{c}\text { Datta et al. } \\
(2012)\end{array}$ \\
\hline $\begin{array}{c}\text { Buscar o desenvolvimento de um sistema que fosse eficiente na } \\
\text { avaliação das práticas ambientais dos fornecedores }\end{array}$ & $\begin{array}{c}\text { Sahu, N. et al. } \\
(2012)\end{array}$ \\
\hline $\begin{array}{c}\text { Abordagens de decisão multicréditos para avaliação e seleção de } \\
\text { fornecedores verdes: uma revisão da literatura }\end{array}$ & $\begin{array}{c}\text { Govindan, et al. } \\
(2013)\end{array}$ \\
\hline $\begin{array}{c}\text { Métodos FAHP, ARAS-F e MSGP integrados para avaliação e seleção } \\
\text { de fornecedores verdes }\end{array}$ & $\begin{array}{c}\text { Liao et al. } \\
(2015)\end{array}$ \\
\hline Avaliando fornecedores verdes de uma perspectiva ambiental verde & $\begin{array}{c}\text { Tsai et al. } \\
(2015)\end{array}$ \\
\hline $\begin{array}{c}\text { Investigar o impacto do tamanho das organizações relacionado à adoção } \\
\text { de práticas de gestão da cadeia de suprimentos na indústria indiana }\end{array}$ & $\begin{array}{c}\text { Vijayvargy, L. } \\
\text { et al. (2016) }\end{array}$ \\
\hline
\end{tabular}
Fonte: Elaborado pelos autores (2017).

Nas últimas décadas, pressões governamentais e sociais sobre as indústrias de componentes fornecedoras da indústria metal mecânica, têm aumentado para melhorarem o desempenho ambiental da cadeia de suprimentos e reduzirem os impactos ambientais (SUSANTY et al.,
2016). Papadopoulos et al. (2014) ressaltam que, considerar estratégias verdes como um diferenciador no mercado, devem estar relacionadas com os comportamentos dos consumidores e da imagem social. Os gestores dessas empresas devem começar a pensar em práticas verdes em todas as etapas de 
produção, reduzindo seus impactos ambientais e aumentando sua eficiência ecológica.

A preocupação ambiental e a sustentabilidade no setor industrial estão em evidencia no panorama mundial, direcionando maiores atenções para as questões que envolvem estes temas. Segundo Sharma et al. (2017), os fornecedores da cadeia de suprimentos que procuram se manter no mercado e prospectar crescimento, passaram a fomentar o aprimoramento das suas responsabilidades ambientais e investir na imagem verde, impulsionando seus negócios. Geralmente, ao se avaliar o desempenho dos fornecedores, as empresas utilizam critérios como qualidade, prazo, preço, por exemplo. Entretanto, a introdução de questões ambientais na GSC passou a ser vista como um diferencial competitivo (VANALLE et al., 2017).

\section{Metodologia}

Iniciou-se com a escolha do tema, por meio da análise nas bases de periódicos (Web of Science e Emerald Insight) por meio das palavras-chave presentes na Figura 1. A partir disso, pelas lacunas encontradas originou-se o problema de pesquisa. Em seguida, foram elencadas as variáveis pertinentes ao estudo. O presente estudo, de caráter exploratóriodescritivo (GIL, 2010), segue as etapas apresentadas na Figura 1.

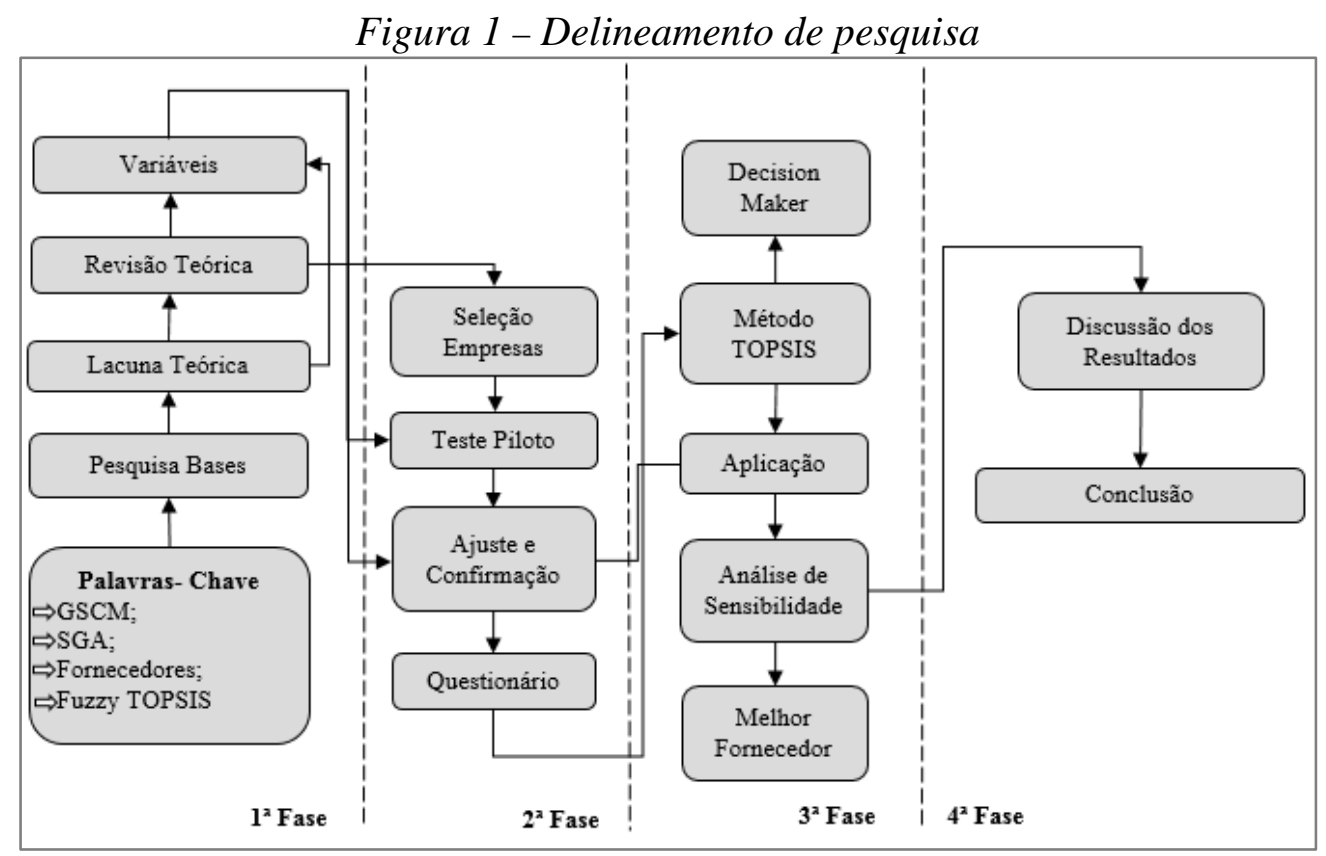

Fonte: Elaborado pelos autores (2017).

Na segunda fase, pré-seleção das empresas em estudo, foi realizado um teste piloto para otimizar a assertividade das escolhas. A partir disso, aplicou-se um questionário para cada gestor, sendo um gestor de produção e outro um supervisor de qualidade, estruturado e formal, desenvolvido com base na literatura. Após a obtenção dos resultados, aplicou-se a modelagem com método fuzzy TOPSIS e, após, análise de sensibilidade. A quarta e última etapa consiste nos resultados e considerações finais.

\subsection{Conjuntos fuzzy e números fuzzy}

Um número fuzzy triangular pode ser representado na forma $\tilde{a}=\left(a_{1}, a_{2}, a_{3}\right)$, sendo que $a_{2}$ evidencia um valor crisp formal para o conjunto fuzzy, $a_{1}$ é o limite inferior e $a_{3}$ é o limite superior. A teoria dos conjuntos fuzzy é caracterizada por uma função de associação $\mu_{\tilde{a}}(x)$ que representa cada elemento através de um número real no intervalo $[0,1]$. A Equação 1 apresenta a definição de números triangulares fuzzy (ZAKERI, 2015). Onde a função de associação $\mu_{\tilde{a}}(x)$ é dada por: 
$\mu_{\tilde{a}}(x)=\left\{\begin{array}{c}0, x \leq a_{1} \\ \frac{x-a_{1}}{a_{2}-a_{1}}, a_{1} \leq x \leq a_{2} \\ \frac{a_{3}-x}{a_{3}-a_{2}}, a_{2} \leq x \leq a_{3} \\ 0, x>a_{3}\end{array}\right.$

no qual $a_{1}, a_{2}$ e $a_{3}$ são números reais e $a_{1}<a_{2}<a_{3}$. $\mathrm{O}$ valor de $x$ em $a_{2}$ apresenta um grau máximo de $\mu_{\tilde{a}}(x)$, como por exemplo, $\mu_{\tilde{a}}(x)=1$. O valor de $x$ em $a_{1}$ apresenta o grau mínimo de $\mu_{\tilde{a}}(x)$, como por exemplo, $\quad \mu_{\tilde{a}}(x)=0$. As constantes $a_{1}$ e $a_{3}$ representam o limite inferior e superior, respectivamente. Essas constantes refletem os atributos de avaliação, em que quanto menor for o intervalo $\left[a_{1}, a_{3}\right]$, maior será a precisão dos dados de avaliação (LIANG, 1999).

\subsection{O método fuzzy TOPSIS}

O fuzzy TOPSIS é uma extensão da técnica TOPSIS usada para resolver problemas de tomada de decisão multicritério MCDM, foi originalmente proposto por Hwang e Yoon (1981), incluindo avaliações difusas de critérios e alternativas (AWASTHI et al., 2010; SHEN et al., 2012). Nesta abordagem, usada amplamente em problemas de tomada de decisão, a alternativa ótima deve ter a menor distância da solução ideal positiva $\left(F P I S^{+}\right)$e a maior distância da solução ideal negativa $\left(F N I S^{-}\right)$. A modelagem completa está presente nos estudos de Awasthi et al. (2010), Shen et al. (2012), Jabbour et al. (2013) e Mangla et al. (2015). As alternativas são classificadas de acordo com o $C C_{i}$ em ordem decrescente. A melhor alternativa será a que estiver mais próxima da FPIS ${ }^{+}$e mais distante da FNIS ${ }^{-}$. Os passos do método (CHEN, 2000; LIMA JUNIOR; CARPINETTI, 2015) são:

Passo 1: Agregar os valores linguísticos fornecidos pelos tomadores de decisão (DMs) em relação à pontuação das alternativas e ao peso dos critérios, utilizada para agregar as pontuações das alternativas;

Passo 2: Construção da matriz de decisão fuzzy $(\widetilde{D})$ para as pontuações e para vetor fuzzy $\widetilde{W}$ para o peso dos critérios, de acordo com as Equações 5 e 6 , respectivamente, onde $\tilde{x}_{i j}, \forall i, j$ e $\widetilde{W}_{j}$ (sendo $i=1,2, \ldots, m ; \quad j=1,2, \ldots, n) \quad$ são $\quad$ variáveis lingüísticas que podem ser descritas por números fuzzy;

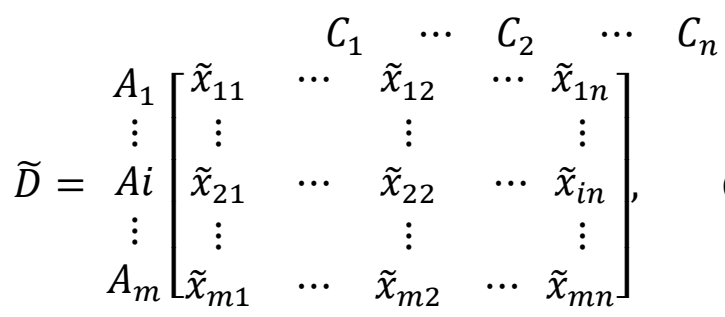

$$
\begin{aligned}
& \widetilde{W}=\left[\widetilde{w}_{1}, \widetilde{w}_{2}, \ldots, \widetilde{w}_{n}\right]
\end{aligned}
$$

Passo 3: Normalizar matriz de decisão fuzzy $\widetilde{D}$, obtida através da Equação 7. Em seguida a matriz normalizada, obtida por meio da multiplicação dos pesos $\left(\widetilde{W}_{j}\right)$ pelos elementos $\left(\tilde{r}_{i j}\right)$;

$\tilde{R}=\left[\tilde{r}_{i j}\right] m x n$

Onde,

$$
i=1,2, \ldots, m ; j=1,2, \ldots, n
$$

$\tilde{r}_{i j}=\left(\frac{a_{i j}}{c_{j}^{+}}, \frac{b_{i j}}{c_{j}^{+}}, \frac{c_{i j}}{c_{j}^{+}}\right), c_{j}^{+}=\max _{i} c_{i j}$

(Critérios de benefício)

$\tilde{r}_{i j}=\left(\frac{a_{j}^{-}}{c_{i j}}, \frac{a_{j}^{-}}{b_{i j}}, \frac{a_{j}^{-}}{a_{i j}}\right), a_{j}^{-}=\min _{i} a_{i j}$

(Critérios de custo)

Passo 4: A matriz normalizada ponderada $(\tilde{V})$ para os critérios é calculada multiplicando os pesos $(\widetilde{w})$ dos critérios com a matriz de decisão fuzzy normalizada $\left(\tilde{r}_{i j}\right)$

$$
\tilde{V}=\left[\tilde{v}_{i j}\right] m x n
$$

$$
i=1,2, \ldots, m ; j=1,2, \ldots, n \text { onde } \tilde{v}_{i j}=\tilde{r}_{i j} \otimes \widetilde{w}_{j}
$$

Passo 5: Definir a solução ideal positiva $\left(\mathrm{A}^{+}\right) \mathrm{e}$ a solução ideal negativa $\left(\mathrm{A}^{-}\right)$, conforme as Equações 8 e 9

$A^{+}=\left(\tilde{v}_{1}^{+}, \tilde{v}_{2}^{+}, \ldots, \tilde{v}_{n}^{+}\right)$onde $\tilde{v}_{j}^{+}=\max _{i}\left\{v_{i j 3}\right\}$

$$
A^{-}=\left(\tilde{v}_{1}^{-}, \tilde{v}_{2}^{-}, \ldots, \tilde{v}_{n}^{-}\right) \text {onde } \tilde{v}_{j}^{-}=\min _{i}\left\{v_{i j 1}\right\}
$$

Passo 6: Calcular a distância $\left(D_{i}^{+}\right)$de cada alternativa de $\mathrm{A}^{+}$(Fuzzy Positive Ideal Solution $F P I S^{+}$) com os valores da matriz $\tilde{R}$, usando a 
Equação 10. E a distância $\left(D_{i}^{-}\right)$de $\mathrm{A}^{-}$(Fuzzy Negative Ideal Solution - FNIS ${ }^{-}$) e os valores da matriz $\tilde{R}$, pela Equação 11, respectivamente.

$$
\begin{aligned}
& d_{i}^{+}=\sum_{j=1}^{n} d_{v}\left(\tilde{v}_{i j}, \tilde{v}_{j}^{+}\right), i=1,2, \ldots, m \\
& d_{i}^{-}=\sum_{j=1}^{n} d_{v}\left(\tilde{v}_{i j}, \tilde{v}_{j}^{-}\right), i=1,2, \ldots, m
\end{aligned}
$$

Ainda, tais equações representam a distância entre dois números fuzzy, que para este artigo foram utilizados números fuzzy triangulares, por apresentarem maior simplicidade de modelagem (CHEN, 2000), podendo ser determinado por meio da Equação 12.

$d(\tilde{a}, \tilde{b})=$

$\sqrt{\frac{1}{3}\left[\left(a_{1}-b_{1}\right)^{2}+\left(a_{2}-b_{2}\right)^{2}+\left(a_{3}-b_{3}\right)^{2}\right]}$
Passo 7: Calcular a proximidade de cada alternativa e classificar as alternativas, e definir ranking de forma descrescentes dos valores de $\mathrm{CC}_{\mathrm{i}}$.

$$
C C_{i}=\frac{d_{i}^{-}}{d_{i}^{-}+d_{i}^{+}}, i=1,2, \ldots, m
$$

Para a avaliação e seleção da melhor alternativa, foram selecionados sete critérios, com base na literatura especifica da área de gestão da cadeia de suprimentos verdes. Foram avaliadas três alternativas potenciais de fornecedores $\left(A_{1}, A_{2}, A_{3}\right)$ para uma indústria metal mecânica. Os critérios e suas referências podem ser visualizados conforme se

\begin{tabular}{|c|c|c|c|}
\hline Critérios & Nome & Definição & Referência \\
\hline $\mathrm{C} 1$ & $\begin{array}{l}\text { Parceiros } \\
\text { ambientais }\end{array}$ & $\begin{array}{l}\text { Relação entre empresa fornecedora e seus } \\
\text { parceiros colaborativos associados no contexto } \\
\text { verde }\end{array}$ & $\begin{array}{l}\text { Shibao, Santos e Moori } \\
\text { (2015); } \\
\text { Ganga, et al. (2016) } \\
\end{array}$ \\
\hline $\mathrm{C} 2$ & $\begin{array}{l}\text { Consumo de } \\
\text { recursos }\end{array}$ & $\begin{array}{l}\text { Consumo de recursos em termos de energia, } \\
\text { água e matéria prima }\end{array}$ & $\begin{array}{l}\text { Cai, et al. (2014); Jennings e } \\
\text { Stadler (2015) }\end{array}$ \\
\hline $\mathrm{C} 3$ & Treinamento & $\begin{array}{l}\text { Treinamento de colaboradores articulando a } \\
\text { aprendizagem das melhores práticas verdes }\end{array}$ & $\begin{array}{l}\text { Jehanzeb e Bashir (2013); } \\
\text { Ford (2014) }\end{array}$ \\
\hline $\mathrm{C} 4$ & Imagem verde & $\begin{array}{l}\text { Produtos ou serviços com ênfase em } \\
\text { sustentabilidade }\end{array}$ & $\begin{array}{l}\text { Agrawal e Das (2014); } \\
\text { Mishra e Sharma (2014); } \\
\text { Polonsky (2015) }\end{array}$ \\
\hline $\mathrm{C} 5$ & \begin{tabular}{|l} 
Politicas de \\
proteção \\
ambiental de \\
fornecedores \\
\end{tabular} & $\begin{array}{l}\text { Refere-se à adesão de leis, regulamentos e } \\
\text { outras politicas / mecanismos de proteção } \\
\text { ambiental }\end{array}$ & $\begin{array}{l}\text { Oliveira (2016); Mendonça, } \\
\text { et al. (2017) }\end{array}$ \\
\hline $\mathrm{C} 6$ & Reciclagem & $\begin{array}{l}\text { É uma reutilização de recursos de forma } \\
\text { eficiente para reduzir o custo de produção e } \\
\text { poluição ambiental }\end{array}$ & $\begin{array}{l}\text { Barbieri (2007); Binnemans, } \\
\text { et al. (2013) }\end{array}$ \\
\hline C7 & $\begin{array}{l}\text { Descarte de } \\
\text { resíduos }\end{array}$ & $\begin{array}{l}\text { Descarte final ou destruição de resíduos, } \\
\text { excesso, sucata, etc., de acordo com a Politica } \\
\text { Nacional de Resíduos Sólidos Lei } \mathrm{n}^{\circ} 12.305 / 10\end{array}$ & $\begin{array}{l}\text { Brasil (2010); Pereira e } \\
\text { Chaves (2015) }\end{array}$ \\
\hline
\end{tabular}
apresenta no Quadro 1.

Quadro 1 - Critérios de seleção e avaliação de fornecedores verdes

Fonte: Elaborado pelos autores (2017).

Cabe destacar, que $C_{2}$ é definido como critério de custo (quanto menor o valor, mais ideal é o fornecedor), no mesmo sentido que, os outros são critérios de benefício (quanto maior o valor, mais ideal é o fornecedor).

Para obtenção dos dados necessários para o método, dois especialistas de uma indústria metal mecânica, sendo um Gerente de produção (DM1) e o outro um Supervisor do setor de compras (DM2), foram orientados a responder um questionário, de acordo com a escala linguística fuzzy apresentada na Tabela 2. 
Tabela 2 - Variáveis linguísticas para avaliar desempenho e importância dos critérios

\begin{tabular}{|c|c|c|c|}
\hline \multicolumn{2}{|c|}{ Importância dos critérios } & \multicolumn{2}{|c|}{ Desempenho dos critérios } \\
\hline Variável linguística & Números fuzzy & Variável linguística & Números fuzzy \\
\hline Muito baixo (MB) & $(1,1,3)$ & Muito ruim (MR) & $(1,1,3)$ \\
\hline Baixo (B) & $(1,3,5)$ & Ruim (R) & $(1,3,5)$ \\
\hline Médio (M) & $(3,5,7)$ & Bom (B) & $(3,5,7)$ \\
\hline Alto (A) & $(5,7,9)$ & Muito bom (MB) & $(5,7,9)$ \\
\hline Muito alto (MA) & $(7,9,9)$ & Ótimo $(\mathrm{O})$ & $(7,9,9)$ \\
\hline
\end{tabular}

Fonte: Elaborado pelos autores (2017).

Um trecho do questionário para avaliação de critérios ambientais é mostrado na Tabela 3. Foram avaliados três potenciais fornecedores para uma indústria metal mecânica, como exemplo ilustrativo do método fuzzy TOPSIS.

Tabela 3 - Questionário para avaliação de critérios ambientais

\begin{tabular}{l|l|l|l|l|l}
\hline \multirow{2}{*}{ Critério } & \multicolumn{4}{l}{ Importância dos critérios } & \multicolumn{1}{l}{} \\
\cline { 2 - 5 } & Muito baixo & Baixo & Médio & Alto & Muito alto \\
\hline Parceiros ambientais & & & & & \\
\hline Consumo de recursos & & & & & \\
\hline Treinamento & & & & & \\
\hline Imagem verde & & & & & \\
\hline Políticas de proteção ambiental de fornecedores & & & & & \\
\hline Reciclagem & & & & & \\
\hline Descarte de resíduos & & & & & \\
\hline
\end{tabular}

Fonte: Elaborado pelos autores (2017).

\section{Resultados e discussões}

A importância de cada um dos fornecedores com relação a cada um dos critérios de avaliação é fornecida com base na percepção dos DMs em relação aos critérios levantados, conforme a Tabela 4.

Tabela 4 - Avaliações linguísticas e pesos agregados fuzzy

\begin{tabular}{|c|c|c|c|c|c|}
\hline \multirow[t]{2}{*}{ Critérios } & \multicolumn{2}{|c|}{ Avaliação linguística dos DM's } & \multicolumn{2}{|c|}{ Pesos dos critérios fuzzy } & \multirow[t]{2}{*}{ Agregado pesos fuzzy } \\
\hline & DM1 & DM2 & DM1 & DM2 & \\
\hline$C_{1}$ & MA & MA & $(7.0,9 \cdot 0,9.0)$ & $(7 \cdot 0,9 \cdot 0,9 \cdot 0)$ & $(7.0,9 \cdot 0,9.0)$ \\
\hline$C_{2}$ & $\mathrm{M}$ & $\mathrm{B}$ & $(3 \cdot 0,5 \cdot 0,7 \cdot 0)$ & $(1.0,3 \cdot 0,5.0)$ & $(1.0,4.0,7.0)$ \\
\hline$C_{3}$ & $\mathrm{~B}$ & $\mathrm{M}$ & $(1 \cdot 0,3 \cdot 0,5.0)$ & $(3 \cdot 0,5 \cdot 0,7 \cdot 0)$ & $(1.0,4.0,7.0)$ \\
\hline$C_{4}$ & MA & A & $(7.0,9 \cdot 0,9.0)$ & $(5.0,7.0,9.0)$ & $(5.0,8 \cdot 0,9.0)$ \\
\hline$C_{5}$ & $\mathrm{~B}$ & B & $(1.0,3.0,5.0)$ & $(1.0,3.0,5.0)$ & $(1.0,3.0,5.0)$ \\
\hline$C_{6}$ & A & A & $(5.0,7.0,9.0)$ & $(5 \cdot 0,7 \cdot 0,9.0)$ & $(5.0,7.0,9.0)$ \\
\hline$C_{7}$ & $\mathrm{~A}$ & MA & $(5.0,7.0,9.0)$ & $(7.0,9 \cdot 0,9.0)$ & $(5.0,8.0,9.0)$ \\
\hline
\end{tabular}

Fonte: Elaborado pelos autores (2017). 
A análise dos resultados obtidos na Tabela 4 evidencia-se que os critérios: Parceiros ambientais $\left(C_{1}\right)$, Imagem verde $\left(C_{4}\right)$ e Descarte de resíduos $\left(C_{7}\right)$, na visão dos DMs, têm maior peso de importância quanto aos critérios estabelecidos. As preocupações da empresa vão ao encontro das definições de Scur e Barbosa (2017), para os autores, deve-se atentar para os fatores que se referem ao uso de materiais reciclados e geração de resíduos tóxicos. Dentro do critério $C_{4}$, por exemplo, os DMs destacam que a imagem verde dos fornecedores pode influenciar no modo que os consumidores veem sua empresa, em vista disso, se busca por empresas que promovam seus produtos ou serviços usando apelos ecológicos, como forma de motivar seus consumidores a adquirir seus produtos ou serviços. De Jesus Pacheco et al. (2016) abordam que o cumprimento de normas ambientais, bem como a divulgação de produtos ambientalmente amigáveis contribuem para que o cliente construa uma imagem de empresa consciente e ecologicamente correta nas suas atividades em geral.

Em seguida, os pesos fuzzy agregados dos fornecedores foram calculados. Nesta etapa, os DMs forneceram suas pontuações, com base nos critérios, para cada alternativa (fornecedor) considerada. Os resultados são apresentados na Tabela 5.

Tabela 5 - Avaliação dos fornecedores pelos DMs

\begin{tabular}{l|c|c|c|c|c|c}
\hline \multirow{2}{*}{ Critérios } & \multicolumn{2}{c}{ Fornecedor A1 } & \multicolumn{2}{c}{ Fornecedor A2 } & \multicolumn{2}{c}{ Fornecedor A3 } \\
\cline { 2 - 7 } & DM1 & DM2 & DM1 & DM2 & DM1 & DM2 \\
\hline C1 & R & R & B & MB & MB & MB \\
\hline C2 & B & R & B & B & B & MB \\
\hline C3 & B & R & R & R & B & R \\
\hline C4 & B & B & R & R & MB & MB \\
\hline C5 & B & MB & R & B & B & B \\
\hline C6 & R & R & B & B & B & MB \\
\hline C7 & R & B & B & B & MB & B \\
\hline
\end{tabular}

Fonte: Elaborado pelos autores (2017).

Após, foram calculados os pesos agregados fuzzy, para cada fornecedor, com base nas respostas dos gestores, conforme apresentado na Tabela 6 .

Tabela 6 - Pesos agregados fuzzy das alternativas

\begin{tabular}{|c|c|c|c|}
\hline Critérios & Fornecedor A1 & Fornecedor A2 & Fornecedor $\mathrm{A} 3$ \\
\hline $\mathrm{C} 1$ & $(1.0,3.0,5.0)$ & $(3.0,6.0,9.0)$ & $(5 \cdot 0,7.0,9.0)$ \\
\hline $\mathrm{C} 2$ & $(1.0,4.0,7.0)$ & $(3 \cdot 0,5 \cdot 0,7.0)$ & $(3.0,6.0,9.0)$ \\
\hline $\mathrm{C} 3$ & $(1.0,4.0,7.0)$ & $(1.0,3 \cdot 0,5.0)$ & $(1.0,4.0,7.0)$ \\
\hline $\mathrm{C} 4$ & $(3.0,5 \cdot 0,7.0)$ & $(1.0,3.0,5.0)$ & $(5.0,7.0,9.0)$ \\
\hline C5 & $(3.0,6.0,9.0)$ & $(1.0,4.0,7.0)$ & $(3.0,5 \cdot 0,7.0)$ \\
\hline C6 & $(1.0,3.0,5.0)$ & $(3.0,5 \cdot 0,7.0)$ & $(3.0,6.0,9.0)$ \\
\hline $\mathrm{C} 7$ & $(1.0,4.0,7.0)$ & $(3.0,5 \cdot 0,7.0)$ & $(3.0,6.0,9.0)$ \\
\hline
\end{tabular}

Fonte: Elaborado pelos autores (2017).

$\mathrm{Na}$ etapa seguinte, a matriz de decisão fuzzy das alternativas é normalizada e ponderada, conforme apresentado na Tabela 7. Aqui, cabe destacar, que o critério $C_{2}$ é calculado como critério de custo e o restante como critérios de benefício. 
Tabela 7 - Alternativas normalizadas e ponderadas, NNIS $^{-}$e FPIS ${ }^{+}$

\begin{tabular}{l|c|c|c|c|c}
\hline \multirow{2}{*}{ Critérios } & \multicolumn{3}{c}{ Matriz de decisão fuzzy normalizada e ponderada } & \multirow{2}{*}{ FNIS $^{-}$} & FNIS $^{+}$ \\
\cline { 2 - 4 } & $A_{1}$ & $A_{2}$ & $A_{3}$ & & \\
\hline$C_{1}$ & $(0.78,3.0,5.0)$ & $(2.33,6.0,9.0)$ & $(3.89,7.0,9.0)$ & 0.78 & 9 \\
\hline$C_{2}$ & $(0.14,1.0,7.0)$ & $(0.11,0.80,2.33)$ & $(0.11,0.67,2.33)$ & 0.11 & 7 \\
\hline$C_{3}$ & $(0.11,0.89,3.89)$ & $(0.11,1.33,3.89)$ & $(0.11,1.78,5.44)$ & 0.11 & 5.44 \\
\hline$C_{4}$ & $(1.67,4.44,7.0)$ & $(0.56,2.67,5.0)$ & $(2.78,6.22,9.0)$ & 0.56 & 9 \\
\hline$C_{5}$ & $(0.33,2.0,5.0)$ & $(0.11,1.33,3.89)$ & $(0.33,1.67,3.89)$ & 0.11 & 5 \\
\hline$C_{6}$ & $(0.56,2.33,5.0)$ & $(1.67,3.89,7.0)$ & $(1.67,4.67,9.0)$ & 0.56 & 9 \\
\hline$C_{7}$ & $(1.67,5.33,9.0)$ & $(1.67,4.44,7.0)$ & $(1.67,5.33,9.0)$ & 1.67 & 9 \\
\hline
\end{tabular}

Fonte: Elaborado pelos autores (2017).

Em seguida, foram obtidas a solução ideal positiva $F P I S^{+}$e a solução ideal negativa $F_{N I S}{ }^{-}$. Os resultados são apresentados nas duas últimas colunas da Tabela 7. As distâncias entre os fornecedores são apresentadas na Tabela 8, representando o espaço entre dois números fuzzy conforme o método vertex, no caso de números fuzzy triangulares.

Tabela 8 - Distância entre fornecedores em relação a cada critério

\begin{tabular}{|c|c|c|c|c|c|c|}
\hline \multirow[t]{2}{*}{ Critérios } & \multicolumn{3}{|c|}{$d_{v}\left(A_{i}, A^{-}\right)$} & \multicolumn{3}{|c|}{$d_{v}\left(A_{i}, A^{+}\right)$} \\
\hline & $A_{1}$ & $A_{2}$ & $A_{3}$ & $A_{1}$ & $A_{2}$ & $A_{3}$ \\
\hline$C_{1}$ & 4,768 & 9,779 & 10,463 & 8,633 & 4,880 & 3,565 \\
\hline$C_{2}$ & 6,947 & 2,328 & 2,292 & 7,188 & 8,720 & 8,815 \\
\hline$C_{3}$ & 3,858 & 3,972 & 5,589 & 5,708 & 5,361 & 4,783 \\
\hline$C_{4}$ & 7,548 & 4,914 & 10,244 & 6,533 & 8,938 & 4,541 \\
\hline$C_{5}$ & 5,244 & 3,972 & 4,089 & 4,032 & 4,759 & 4,428 \\
\hline$C_{6}$ & 4,781 & 7,278 & 9,408 & 9,177 & 6,932 & 6,058 \\
\hline$C_{7}$ & 8,194 & 6,009 & 8,194 & 5,601 & 6,533 & 5,601 \\
\hline$\Sigma$ & 41,341 & 38,251 & 50,279 & 46,872 & 46,122 & 37,791 \\
\hline
\end{tabular}

Fonte: Elaborado pelos autores (2017).

A maior distância identificada a partir dos resultados obtidos na Tabela 8 é da alternativa $A_{3}$, cuja se mostra $21 \%$ mais distante da solução ideal negativa e $24 \%$ mais próxima da solução ideal positiva, quando comparada com a alternativa $A_{1}$. A solução ótima deve estar mais próxima da solução ideal positiva e mais distante da solução ideal negativa (Uygun e Dede, 2016). Finalmente, combinando a diferença entre as distancias, obtém-se o coeficiente de proximidade $C C_{i}$. Os resultados são apresentados na Tabela 9.
Tabela 9 - Coeficiente de proximidade $\left(C C_{i}\right)$.

\begin{tabular}{l|l|l|l}
\hline & \multicolumn{3}{|l}{ Fornecedores } \\
\hline & $\mathrm{A}_{1}$ & $\mathrm{~A}_{2}$ & $\mathrm{~A}_{3}$ \\
\hline$d_{i}^{-}$ & 41,341 & 38,251 & 50,279 \\
\hline$d_{i}^{+}$ & 46,872 & 46,122 & 37,791 \\
\hline$C C_{i}$ & 0,469 & 0,453 & 0,571 \\
\hline \multicolumn{4}{|c}{ Fonte: Elaborado pelos autores (2017). }
\end{tabular}

Comparando os resultados das três alternativas, por meio dos valores de $C C_{i}$, destaca-se que, em ordem decrescente, o ranking dos fornecedores é $A_{3}>A_{1}>A_{2}$. É possível identificar 
que o fornecedor $A_{3}$ apresentou o maior desempenho global entre as alternativas. Isto significa que é o fornecedor mais próximo da solução ideal positiva $\left(F P I S^{+}\right)$e mais distante da solução ideal negativa $\left(F N I S^{-}\right)$, confirmando as definições apresentadas por Uygun e Dede (2016). Na análise dos dados em relação a ponderação dos pesos pelos DMs, o fornecedor $A_{3}$ é o que apresenta maior afinidade com os critérios ambientais analisados, sobretudo, nos critérios $C_{1}, C_{4}, C_{6}$ e $C_{7}$, onde verificou-se que são fatores que podem definir pela contratação ou não de determinado fornecedor.

\subsection{Análise de sensibilidade}

Aplicou-se a análise de sensibilidade para ratificar as respostas encontradas no desenvolvimento do método. Dessa forma, foi investigado o impacto do peso dos critérios sobre a seleção de fornecedores verdes. Para isso, os pesos de cada um dos critérios foram alternados, resultando em 7 combinações, conforme detalhado na Tabela 10.

Tabela 10 - Experimentos da análise de sensibilidade

\begin{tabular}{l|l|c|c|c|c}
\hline Experimento & Definição & $A_{1}$ & $A_{2}$ & $A_{3}$ & Ranking \\
\hline E1 & $C 1=(3.0,5.0,7.0), C 2-C 7=(1.0,4.0,7.0)$ & 0,351 & 0,318 & 0,396 & $A_{3}>A_{1}>A_{2}$ \\
\hline E2 & $C 2=(5.0,7.0,9.0), C 1, C 3-C 7=(5.0,7.0,7.0)$ & 0,415 & 0,375 & 0,480 & $A_{3}>A_{1}>A_{2}$ \\
\hline E3 & $C 3=(7.0,9.0,9.0), C 1, C 2-C 7=(1.0,4.0,7.0)$ & 0,363 & 0,332 & 0,412 & $A_{3}>A_{1}>A_{2}$ \\
\hline E4 & $C 4=(1.0,4.0,7.0), C 1, C 3-C 7=(7.0,7.0,9.0)$ & 0,454 & 0,426 & 0,519 & $A_{3}>A_{1}>A_{2}$ \\
\hline E5 & $C 5=(5.0,8.0,9.0), C 1, C 4-C 7=(5.0,7.0,9.0)$ & 0,476 & 0,425 & 0,518 & $A_{3}>A_{1}>A_{2}$ \\
\hline E6 & $C 6=(7.0,9.0,9.0), C 1, C 5-C 7=(5.0,8.0,9.0)$ & 0,489 & 0,457 & 0,568 & $A_{3}>A_{1}>A_{2}$ \\
\hline E7 & $C 7=(3.0,5.0,5.0), C 1-C 6=(5.0,7.0,9.0)$ & 0,439 & 0,407 & 0,508 & $A_{3}>A_{1}>A_{2}$ \\
\hline
\end{tabular}

Fonte: Elaborado pelos autores (2017).

Nota-se que, entre as 7 simulações realizadas, a alternativa $A_{3}$ obteve a maior pontuação em todas, corroborando $\mathrm{O}$ resultado encontrado no desenvolvimento do método fuzzy TOPSIS. Entretanto, não se pode concluir que o peso dos critérios não influencia na tomada de decisão, haja vista que, mais simulações, variando o peso de importância dos critérios, poderiam ter sido realizadas. Porém, por uma limitação de pesquisa, foram consideradas 7 variações aleatórias de peso. Sendo assim, em cada experimento variou-se o peso para cada um dos critérios, definindo como mais alto (7.0,9.0,9.0) até o mais baixo (1.0,4.0,7.0). Os resultados da análise de sensibilidade são apresentados na Figura 2.

Figura 2 - Resultados da análise de sensibilidade.

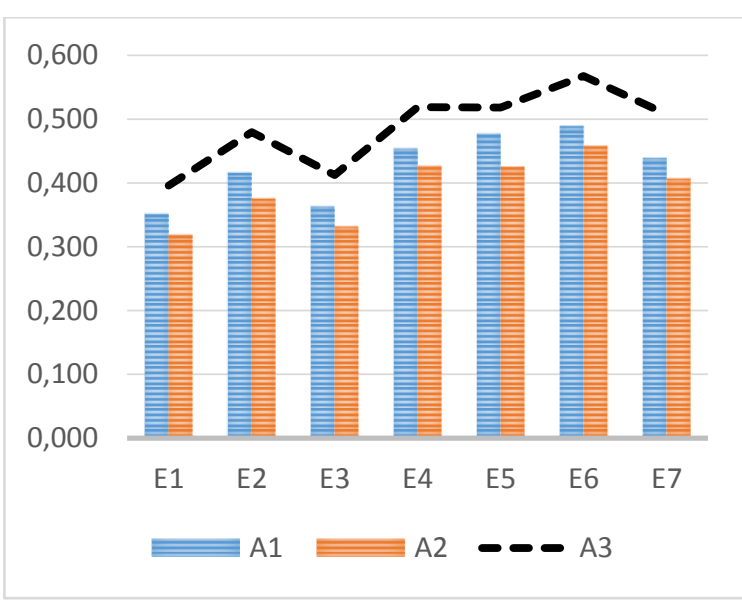

Fonte: Elaborado pelos autores (2017). 
Pode ser observado que, nas 7 simulações realizadas, o fornecedor $A_{3}$ perpassa as pontuações dos fornecedores $A_{1}$ e $A_{2}$. Isto significa que, dos 7 critérios que foram levantados, com base nas práticas da GSCM, o fornecedor $A_{3}$ apresentou um equilíbrio considerável, no que se refere ao seu desempenho ambiental, demonstrando que atende, de maneira satisfatória, a maioria dos critérios analisados, especialmente, parceiros ambientais $\left(C_{1}\right)$, imagem verde $\left(C_{4}\right)$, reciclagem $\left(C_{6}\right)$ e descarte de resíduos $\left(C_{7}\right)$. Por outro lado, os fornecedores $A_{1}$ e $A_{2}$, embora não tenham apresentado desempenho global acima de seu concorrente, nos critérios políticas de proteção ambiental $\left(C_{5}\right)$ e parceiros ambientais $\left(C_{1}\right)$, demonstraram possuir bom desempenho.

O efeito positivo de ações ambientais na gestão da cadeia de suprimentos e no desempenho operacional é evidenciado no desenvolvimento de algumas pesquisas, em diferentes seguimentos industriais (Awasthi et al., 2010; Jabbour et al., 2013). A nível estratégico da organização, esta pesquisa mostra que a preocupação com as questões relacionadas a sustentabilidade da cadeia de fornecimento, tem refletido em ações mais proativas dos gestores, buscando reduzir os impactos ambientais de suas operações.

\section{Conclusões}

A GSCM no Brasil possui um campo fértil para a pesquisa, pois ainda é considerado um assunto carente de atenção com intuito de alcançar menores índices de impactos ao meio ambiente, embora que, a literatura referente a fornecedores seja opulenta, as pesquisas no país que abordam critérios ambientais para seleção de fornecedores verdes ainda são limitadas. Nesse sentido, devido a pressões governamentais, as empresas estão direcionando esforços para o cumprimento de legislações ambientais, assim como adotar práticas verdes que o mercado competitivo também exige. A adoção de inciativas verdes possibilita o surgimento de vantagens competitivas para as empresas, no entanto, para alcançar a estabilidade no mercado, os fornecedores necessitariam de treinamentos para adequarem seus processos e produções a este novo conceito de mercado quanto à adoção de práticas verdes na gestão da cadeia de suprimentos.
Esta pesquisa propôs como objetivo avaliar o desempenho ambiental de três potenciais fornecedores por meio do método de tomada de decisão multicritério fuzzy TOPSIS e selecionar a melhor alternativa. Os critérios selecionados para o estudo, foram propostos de acordo com a literatura pesquisada em GSCM. A lacuna de pesquisa fundamentou-se na incerteza e na falta de respostas quantitativas a respeito da avaliação do desempenho ambiental de fornecedores de uma empresa Metalmecânica do Sul brasileiro.

Em virtude da relação da adoção de inciativas verdes com o nível de desempenho, como resultado, o estudo evidencia que as empresas estão em amadurecimento na adoção de práticas verdes na cadeia de suprimentos, evidenciando que, embora o fornecedor $A_{3}$ tenha apresentado o melhor desempenho global, os fornecedores $A_{1}$ e $A_{2}$ também estão adotando, gradativamente, ações sustentáveis em suas cadeias de suprimentos. No entanto, quanto à relação entre a GSCM e o desempenho operacional dos processos, o estudo não é conclusivo para comprovação.

O estudo apresenta três limitações constatadas, a primeira remete-se ao desenvolvimento da pesquisa em uma única empresa do setor metal mecânico. A segunda, por não expressar a cultura ambiental dos fornecedores em relação à cadeia de suprimentos, e a terceira, por tratar-se de fatores linguísticos através de escalas, possibilitando o aparecimento de subjetividade nas respostas. $\mathrm{Na}$ busca do fortalecimento da pesquisa, sugere-se o refinamento do estudo levando em consideração a cultura ambiental dos fornecedores, assim como replicar o estudo em um número maior de empresas do mesmo setor. Além disso, o aprofundamento acerca das variáveis utilizadas e a replicação do modelo em outros segmentos industriais podem contribuir para mais robustez de trabalhos futuros. Ademais, outras técnicas como, metodologia do sistema cinza e Analysis Hierarchy Process, podem aumentar a precisão dos resultados. 


\section{Referências}

AGRAWAL, A. D.; DAS, M. Green Marketing: Sustainable marketing strategy. Indira Management Review, v. 17, 2013.

AWASTHI A, CHAUHAN SS, GOYAL SK (2010) A fuzzy multicriteria approach for evaluating environmental performance of suppliers. International Journal of Production Economics. 126: 370-378.

BARBIERI, J. C. Organizações inovadoras sustentáveis. Caderno de Inovação, v. 3, p. 5-9, 2007.

BINNEMANS, K. et al. Recycling of rare earths: a critical review. Journal of Cleaner Production, v. 51, p. 1-22, 2013.

BRASIL. LEI N ${ }^{\circ} 12.305$, de 2 de agosto de 2010. Institui a Política Nacional de Resíduos Sólidos; altera a Lei no 9.605, de 12 de fevereiro de 1998; e dá outras providências. 2010. Disponível: <http://www.planalto.gov.br/ccivil_03/_ato2007 -2010/2010/lei/112305.htm>. Acesso em: 18 jun. 2017.

CAI, L. X. et al. Sustainability analysis and resource management for wireless mesh networks with renewable energy supplies. IEEE Journal on Selected Areas in Communications, v. 32, n. 2, p. 345-355, 2014.

CHEN, C. Extensions of the TOPSIS for group decision-making under fuzzy environment. Fuzzy sets and systems, v. 114, n. 1, p. 1-9, 2000.

CHEN C.T.; LIN, C.T.; HUANG, S. F.; A fuzzy approach for supplier evaluation and selection in supply chain management. International Journal of Production Economics, 102:289-301, 2006.

CHEN, C.C.; TSENG, Y.H; LIN, Z.S. Lin Implementation of green supply chain management in uncertainty. International Conference on IEEM, IEEE 7-10 Dec, p. 260264. 2010.
CHIOU, C.Y.; HSU, C.W.; HWANG, W.Y. Comparative investigation on green supplier Selection of the American, Japanese and Taiwanese electronics industry in China. International Conference on IE\&EM, IEEE 8-11 Dec (2008), pp. 1909-1914.

DATTA, S.; SAMANTRA, C.; MAHAPATRA, S. S.; BANERJEE, S.; BANDYOPADHYAY, A. Green supplier evaluation and selection using VIKOR method embedded in fuzzy expert system with interval-valued fuzzy numbers. International Journal of Procurement Management, v. 5, n. 5, p. 647-678, 2012.

CARVALHO, A. P. de; BARBIERI, J. C.; Inovações socioambientais em cadeias de suprimento: um estudo de caso sobre o papel da empresa focal. RAI Revista de Administração e Inovação, v. 10, n. 1, p. 232-256, 2013.

PACHECO, D. A. de J.; ANTUNES JÚNIOR, J. A.; JUNG, C. F.; LUZ, D. F. da; PERGHER, I. (2016). Práticas de sustentabilidade em cadeias de suprimentos. Interciencia, 41: 506-511

DUBEY, R.; GUNASEKARAN, A.; PAPADOPOULOS, T.; CHILDE, S. J.; Green supply chain management enablers: Mixed methods research. Sustainable Production and Consumption, v. 4, p. 72-88, 2015.

EL HOUR S.; AOUANE M.; CHAOUCH A.; Perception of Industrial Enterprise about the Management of Environmental Impacts towards to Standards ISO 14001: 2015: The Case of the Industrial Companies of Casablanca-Settat Region Morocco. Environmental Science: An Indian Journal, 12 11. 2016.

FORD, J. K. Improving training effectiveness in work organizations. Psychology Press, 2014.

GANGA, G. M. D. et al. Métodos quantitativos para seleção de fornecedores sustentáveis: uma revisão sistemática da literatura. Revista Produção Online, v. 16, n. 4, p. 1434-1457, 2016. 
GIL, A. C. Como elaborar projetos de pesquisa. -5. ed. - São Paulo: Atlas, 2010.

GOVINDAN K.; KALIYAN M.; KANNAN D.; HAQ A.N. Barriers analysis for green supply chain management implementation in Indian industries using analytic hierarchy process. International Journal of Production Economics, v. 147 , p. 555-568. 2014.

GRISI, R.M.; GUERRA, L.; NAVIGLIO, G. Supplier performance evaluation for green supply chain management. Business Performance Measurement and Management, v. 4, pp. 149-163. 2010.

GUPTA, H.; BARUA, M. K.; Supplier selection among SMEs on the basis of their green innovation ability using BWM and fuzzy TOPSIS. Journal of Cleaner Production, 2017

HWANG C.L.; YOON K.; Multiple Attribute Decision Making. Berlin: Springer-Verlag; 1981.

JABBOUR ABL, DE SOUZA AZEVEDO F, ARANTES AF, JABBOUR CJC. Greening the Supply Chain: Some Evidence from Companies Located in Brazil. Gestão \& Produção. 20: 953962. 2013.

JEHANZEB, K.; BASHIR, N. A. Training and development program and its benefits to employee and organization: A conceptual study. Training and Development, v. 5, n. 2, 2013.

JENNINGS, B.; STADLER, R. Resource management in clouds: Survey and research challenges. Journal of Network and Systems Management, v. 23, n. 3, p. 567-619, 2015.

JUNIOR, F. R. L.; OSIRO, L.; CARPINETTI, L. C. R.; A comparison between Fuzzy AHP and Fuzzy TOPSIS methods to supplier selection. Applied Soft Computing, v. 21, p. 194-209, 2014.

KHALILI, N. R.; DUECKER, S. Application of multi-criteria decision analysis in design of sustainable environmental management system framework. Journal of Cleaner Production, v. 47, p. 188-198, 2013.
KUEI, C. H.; MADU, C. N.; CHOW, W. S.; CHEN, Y.; Determinants and associated performance improvement of green supply chain management in China. Journal of Cleaner Production, v. 95, p. 163-173, 2015

LIANG GS (1999) Fuzzy MCDM based on ideal and anti-ideal concepts. European Journal of Operational Research. 112:682-691

LIAO, CHIN-NUNG; FU, YAN-KAI; WU, LICHUN. Integrated FAHP, ARAS-F and MSGP methods for green supplier evaluation and selection. Technological and Economic Development of Economy, v. 22, n. 5, p. 651669, 2016.

LIMA JUNIOR, F. R.; CARPINETTI, L. C. R. Uma comparação entre os métodos TOPSIS e FuzzyTOPSIS no apoio à tomada de decisão multicritério para seleção de fornecedores. Gestão \& Produção, v. 22, p. 17-34, 2015

LUTHRA, S.; KUMAR, S.; GARG, D.; HALEEM, A.; Comparative evaluation of GSCM practices in automotive components manufacturing firms of India: a fuzzy TOPSIS approach. International Journal of Logistics Systems and Management, v. 25, n. 3, p. 358-390, 2016.

MANGLA SK, KUMAR P, BARUA MK (2015) Prioritizing the responses to manage risks in green supply chain: An Indian plastic manufacturer perspective. Sustainable Production and Consumption. 1: 67-86

MAZZI, A.; TONIOLO, S.; MASON, M.; AGUIARI, F.; SCIPIONI, A.; What are the benefits and difficulties in adopting an environmental management system? The opinion of Italian organizations. Journal of Cleaner Production. 139: 873-885(2016)

MENDONÇA, J. C. A., et al. Logística reversa no brasil: um estudo sobre o mecanismo ambiental, a responsabilidade social corporativa e as legislações pertinentes. Revista Capital Científico-Eletrônica (RCCe)-ISSN 2177-4153, v. 15, n. 2, p. 130-147, 2017. 
MISHRA, P.; SHARMA, P. Green marketing: Challenges and opportunities for business. BVIMR Management Edge, v. 7, n. 1, 2014.

OLIVEIRA, E. C. Influência da variável ambiental no processo de escolha dos fornecedores: Um Estudo em Agroindústrias da Microrregião de Assis-SP. Revista Produção e Desenvolvimento, v. 2, n. 2, p. 77-100, 2016.

PAPADOPOULOS, I.; KARAGOUNI, G.; TRIGKAS, M.; BELTSIOU, Z.; Mainstreaming green product strategies: Why and how furniture companies integrate environmental sustainability? EuroMed Journal of Business. 9: 293-317 (2014)

PEREIRA, B. M.; CHAVES, G. L. D. A implantação da política nacional de resíduos sólidos (PNRS) no estado do Espírito Eanto. Brazilian Journal of Production Engineering-BJPE, v. 1, n. 1, p. 1-3, 2015.

SAHU, N. K.; DATTA, S.; SANKAR MAHAPATRA, S. Establishing green supplier appraisement platform using grey concepts. Grey Systems: Theory and Application, v. 2, n. 3, p. 395-418. 2012.

SCUR, G.; BARBOSA, M. E. Green supply chain management practices: Multiple case studies in the Brazilian home appliance industry. Journal of Cleaner Production. 141: 1293-1302. 2017.

SCUR, G.; BARBOSA, M. E.; Green supply chain management practices: Multiple case studies in the Brazilian home appliance industry. Journal of Cleaner Production, v. 141, p. 1293-1302, 2017

SHARMA, V. K.; CHANDNA, P.; BHARDWAJ, A.; Green supply chain management related performance indicators in agro industry: A review. Journal of Cleaner Production, v. 141, p. 1194-1208, $2017 . \quad$ doi: 10.1016/j.jclepro.2016.09.103

SHEN L, OLFAT L, GOVINDAN K, KHODAVERDI R, DIABAT A (2012) A fuzzy multi criteria approach for evaluating green supplier's performance in green supply chain with linguistic preferences. Resources, Conservation and Recycling. 74: 170-179

SHEN, L.; OLFAT, L.; GOVINDAN, K.; KHODAVERDI, R.; DIABAT, A.; A fuzzy multi criteria approach for evaluating green supplier's performance in green supply chain with linguistic preferences. Resources, Conservation and Recycling, v. 74, p. 170-179, 2013.

SHIBAO, F. Y.; SANTOS, M. R.; MOORI, R. G. Gestão da cadeia de suprimentos verde: uma comparação entre Brasil, China e Japão. Gestão Contemporânea, n. 16, 2015.

SUSANTY, A.; SARI, D. P.; BUDIAWAN, W.; KURNIAWAN, H. Improving Green Supply Chain Management in Furniture Industry Through Internet Based Geographical Information System for Connecting the Producer of Wood Waste with Buyer. Procedia Computer Science, v. 83, p. 734-741, 2016.

TSAI, S. B.; WEI, Y. M.; CHEN, K. Y.; XU, L.; DU, P.; LEE, H. C. Evaluating green suppliers from a green environmental perspective. Environment and Planning B: Planning and Design, v. 43, n. 5, p. 941-959, 2016.

TYAGI M, KUMAR P, KUMAR D (2015) Parametric selection of alternatives to improve performance of green supply chain management system. Procedia-Social and Behavioral Sciences. 189: 449-457

UYGUN, Ö.; DEDE, A. Performance evaluation of green supply chain management using integrated fuzzy multi-criteria decision making techniques. Computers \& Industrial Engineering, v. 102, p. 502-511, 2016.

VANALLE, R. M.; GANGA, G. M. D.; GODINHO FILHO, M.; LUCATO, W. C.; Green supply chain management: An investigation of pressures, practices, and performance within the Brazilian automotive supply chain. Journal of Cleaner Production, v. 151, p. 250-259, 2017. doi: 10.1016/j.jclepro.2017.03.066. 
VIJAYVARGY, L.; THAKKAR, J.; AGARWAL, G. Green supply chain management practices and performance: the role of firm-size for emerging economies. Journal of Manufacturing Technology Management, v.28, n.3. 2017.

ZADEH, L. A. Fuzzy sets. Information and Control ; 8:338-53, 1965.

ZAKERI, S.; KERAMATI, M. A. Systematic combination of fuzzy and grey numbers for supplier selection problem. Grey Systems: Theory and Application. 5: 313-343.2015 
